\title{
The use of titanium dental implants vs zirconium dioxide dental implants in esthetic zone. Systematic review.
}

Obando, JC. ${ }^{1}$, Marques, D. 1,2, Caramês J.2, Manrique C. ${ }^{1}$, Santiago J. ${ }^{1}$, Trapote S. ${ }^{1}$, Jiménez J. ${ }^{1}$ 1- Universidad Europea Madrid, Madrid, Spain

2- Faculty of Dental Medicine, Lisbon University, Portugal

\section{Abstract}

Background: The use of zirconium dental implants for esthetic purposes seems to have a promising future due to similar survival rates to titanium implants. Other added mentioned proprieties are white color, the small level of bacterial adherence

Aim/Hypothesis: The main aim of this systematic review was to evaluate the zirconium oxide implants success and survival rates ( $\mathrm{ZrO} 2)$ versus the titanium implants $(\mathrm{Ti})$ in the esthetic area.

Materials and Methods: This Systematic review was registered at PROSPERO with the reference CRD42015019546. Clinical trials that evaluated zirconium oxide implants were searched in seven electronic databases (PubMed, Lilacs, Science Direct, Cochrane Collaboration, Evidence Based Dentistry (EBD), Journal of were included according to pre-established inclusion criteria. A manual search was performed on the bibliography of the collected articles, and the authors were contacted for additional references. Success and survival rate for titanium implants (Ti) was compared with the zirconium oxide implants success and survival rate (ZrO2). The quality of the included articles was evaluated using the Critical Appraisal Skills Programme (CASP) criteria, thus being analyzed at both study and outcome level.

Results: Seventeen articles were included and the results obtained after calculating the data from each study, in which the zirconium oxide dental implants (ZrO2) location (maxilla or mandible, anterior or posterior) is described. Number of Mean: 32 months (2.6 years). In order to analyze the information about the esthetic area, the studies which did not describe the placement of the zirconium oxide implants in the anterior area (ZrO2) were discarded, only implants placed from 14 to 24 and 34 to 44 (esthetic area) were accounted for: 486 ZrO2 implants. Of the included studies only two were randomized controlled trials; twelve prospective trials and three retrospective studies. Several methodological limitations on the collected articles and debated in this review. The overall survival rate of the zirconium oxide implants of one and two pieces resulted in $95 \%$.

Conclusions and Clinical implications: Although the available scientific evidence base is at considerable risk of bias, it is possible to conclude that zirconia dental implants could be used in esthetic zone with $95 \%$ survival. The different study designs and methodology make difficult to present an overal conclusion regarding the efficacy of $\mathrm{ZrO} 2$ implants in maintaining crestal bone levels and periimplant soft tissue health. Further long-term, randomized controlled trials with a standard methodology are needed.

\section{Background and Aim}

This systematic review intends to answer the following PICO question: "In the edentulous anterior maxilla or mandible (esthetic zone) of healthy patients the use of zirconium oxide implants compared to titanium implants presents better success and survival rates"

\section{Methods and Materials}

\section{Electronic Search Strategies.}

An electronic search was carried out in the following databases: PubMed, Evidence Based Dentistry, Journal of Evidence-Based Dental Practice, Lilacs, Science Direct, Cochrane Collaboration, NH Evidence. The search strategy was adapted to each database following the guidelines used for Medline search. Briefly, PubMed was used to identify the Medical Subject Headings (MeSH) terms corresponding to each term. Identification of the MeSH terms: "zirconia", "zirconio", "ceramics" "zirconium oxide", "dental implants" and boolean operators were used: AND, OR, NOT.

Additional Strategies and Other Resources:

A manual search was conducted by checking all the references of the studies considered relevant and possibly relevant at the end of the first stage. Additionally the following journals were hand-searched for potentially relevant studies. Clinica Implant Dentistry and Related Research, International Journal of Oral \& Maxillofacial Implants, Journal of Oral Implantology, Clinical Oral Implants Research, Journal of Clinical Periodontology and Journal of Periodontology.

The corresponding authors of the included studies were contacted via email and of additional works of the sam aur number of implants placed and lost in the esthetic area (pre-molar to pre-molar).

Criteria in the Selection of Studies.

Studies published in english, spanish and portuguese published between 2006 and 2016 that were meta-analyses, systematic reviews, randomised clinical trials, controlled clinical trials, pragmatic clinical trials, clinical trials and cohort studies were considered. The inclusion criteria were: studies with zirconia dental implants with a minimum follow up period of 6 months in which implant survival or success rate was assessed. Additional data if available, was retrieved, mainly, location of (immediate/delayed), crestal bone loss (CBL), temporization mode procedures during surgery (yes/no), implant design ( 1 piece / 2 piece) and plaque index (PLI) (percentage, index).

Data Collection and Quality Assessment.

The selection of each study followed an assessment in three phases according PRISMA guidelines.

All studies considered relevant in the previous phases were subjected to a critical assessment of their scientific merit according to the questionnaires of the Critica Appraisal Skills Program (CASP) of the Public Health Resource Unit (2006) their counterpart evaluations performed independent assessments. On completion the evaluations discrepancies were resolved by mutual accordance.

Studies with more than half of the questions in the CASP questionnaire rated as negative were considered to have a high risk of bias. Therefore only the trials whose CASP evaluations were equal or greater than $50 \%$ were selected for the final analysis in this review.

\section{Results}

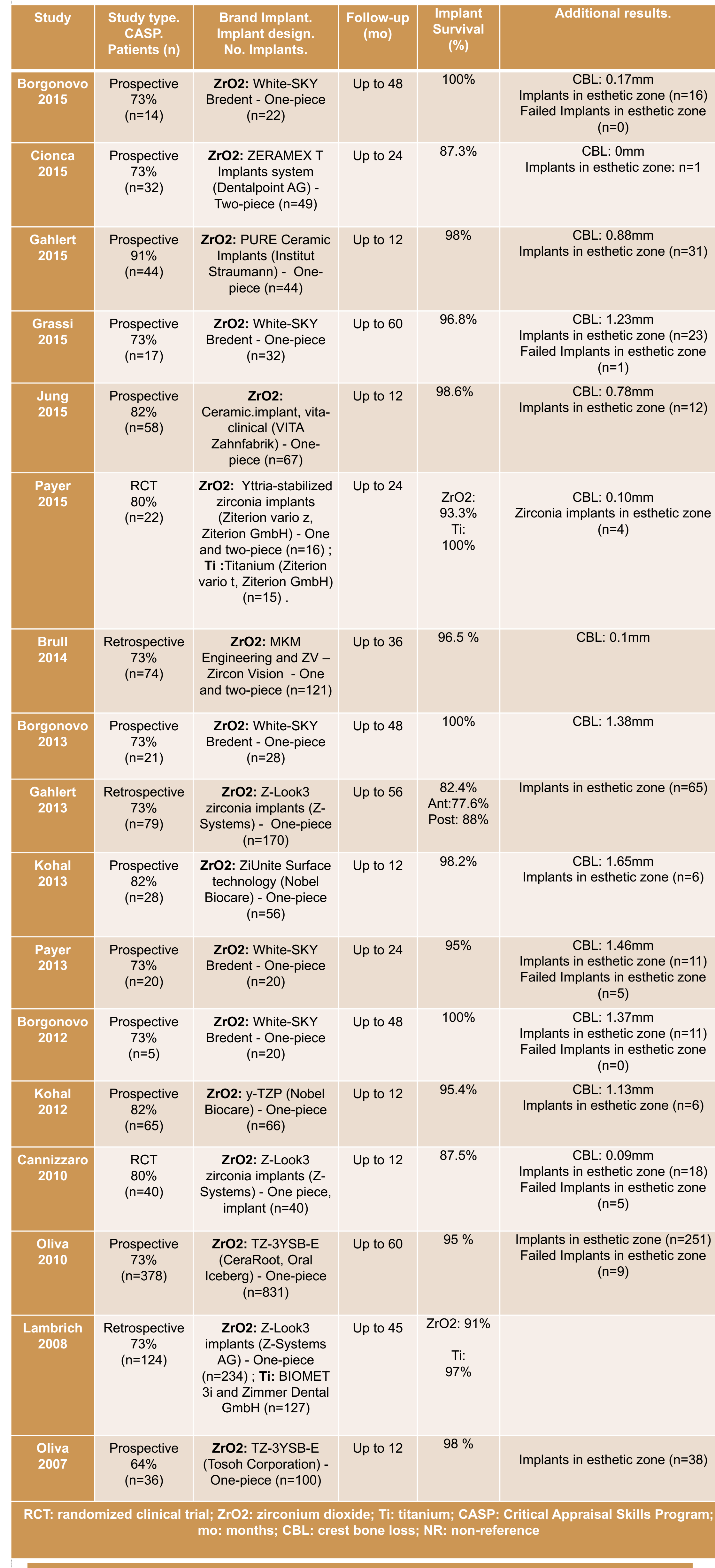

\section{Conclusions}

Although the available scientific evidence is limited and at considerable risk of bias, it is still possible to interpret the information that the zirconia dental implan could be used with $95 \%$ survival which is comparable to titanium dental implants survival.

The information regarding specific outcomes in the esthetic zone are scarce and due to different study designs and methodology it was not possible to achieve clear conclusion regarding the obtained data.

Further long-term, randomized controlled trials with a standard methodology are needed.

\section{References}

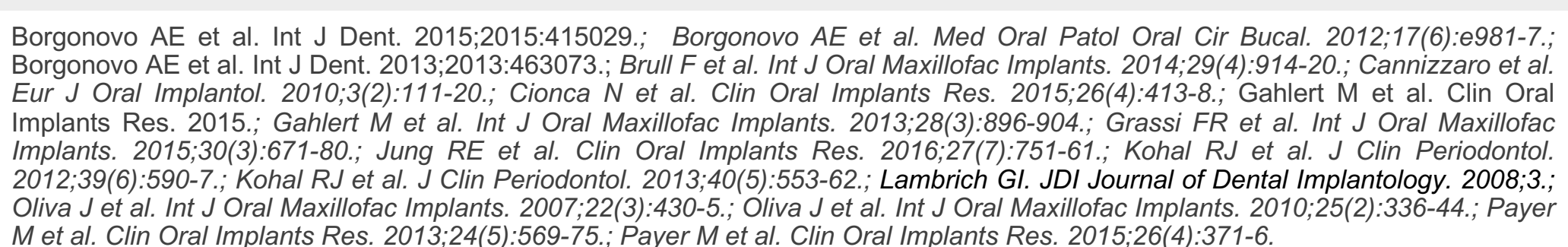

